\title{
Dorsal: Ferramenta para Geração de Modelos de Dados para Aplicações voltadas a Saúde e Cuidado de Idosos
}

\author{
Pedro Oliveira ${ }^{1}$, Italo Araújo ${ }^{1}$, Evilasio Junior ${ }^{1}$, Paulo Duarte ${ }^{1}$, Ismayle S. Santos ${ }^{1}$, \\ Rossana M. C. Andrade ${ }^{1}$, Ivana Cristina H. C. Barreto ${ }^{2}$ e Luiz Odorico M. Andrade ${ }^{2}$ \\ ${ }^{1}$ Grupo de Redes de Computadores, Engenharia de Software e Sistemas (GREat) \\ Universidade Federal do Ceará (UFC) - Fortaleza - CE - Brasil \\ ${ }^{2}$ Fundação Oswaldo Cruz - Fortaleza - CE - Brasil \\ \{pedromartins, italoaraujo, evilasiojunior, pauloduarte, ismaylesantos, \\ rossana\}egreat.ufc.br, \{ivana.barreto, odorico.monteiro\}efiocruz.br
}

\begin{abstract}
The aging population, combined with a greater interest in health and well-being, has motivated the development of web and mobile applications to provide longevity with a better quality of life. However, since data modeling for elderly health systems is a complex task, in previous work, we created a taxonomy for 7 different types of elderly health systems to help developers to deal with this difficulty. This taxonomy is composed of 21 categories and 87 characteristics that organize the information handled by these systems. For example, there is the "Glucose Control" category with two characteristics, date and glycemia. However, the combination of types of applications, categories, and characteristics generates a large number of possibilities, making it difficult to use the taxonomy. So, the Dorsal tool, proposed in this paper, aims to create data models in Java or Javascript from this taxonomy to reduce the effort in the development of elderly health applications.
\end{abstract}

Resumo. $O$ envelhecimento da população aliado a um maior interesse por saúde e bem-estar têm motivado o desenvolvimento de aplicações web e mobile para prover longevidade com maior qualidade de vida. Contudo, modelar dados para sistemas de saúde do idoso é uma tarefa complexa e, então, para auxiliar desenvolvedores a lidar com essa dificuldade, em um trabalho anterior do nosso grupo de pesquisa, foi desenvolvida uma taxonomia de dados para 7 tipos de sistemas de saúde do idoso. Essa taxonomia é composta por 21 categorias e 87 características que organizam os dados que devem ser manipulados por esses sistemas. Por exemplo, a categoria “Controle de Glicose” (Glucose Control) possui data e glicemia como duas características. Entretanto, a combinação entre os tipos de aplicação, categorias e características gera um grande número de possibilidades, dificultando a utilização desta taxonomia. Sendo assim, a ferramenta Dorsal, proposta neste artigo, tem como objetivo gerar modelos de dados em Java ou Javascript a partir desta taxonomia para reduzir o esforço de desenvolvimento de aplicações para saúde do idoso.

Link do Vídeo: https://great-ufc.github.io/dorsal/video

\section{Introdução}

O envelhecimento populacional é um fenômeno que está ocorrendo no mundo todo. De acordo com a Organização Mundial da Saúde (OMS) [Robbins et al. 2018], o número de 
idosos no mundo vai praticamente dobrar em 2050, passando a ser $22 \%$, quando comparado ao ano de 2015, que era 12\% [WHO 2007]. Esses dados sugerem a necessidade da criação de meios para auxiliar no processo de envelhecimento, o que, ainda de acordo com a OMS, pode ser realizado com o apoio de aplicativos.

Embora existam diversas aplicações voltadas para a saúde de idosos, ainda persiste a dificuldade de sistematizar as informações a serem manipuladas por tais aplicações [Woll and Bratteteig 2019]. Por exemplo, sistemas epidemiológicos requerem dados detalhados do perfil do idoso, como o registro de vacinação, para dar suporte à gestão de epidemias. Já em sistemas focados no monitoramento de saúde em ambientes específicos, como a casa do idoso, os dados de registro de vacinação não são essenciais.

Quanto à organização dos dados, em um trabalho anterior foi proposta uma taxonomia [Araújo et al. 2020] que auxilia na seleção de dados relevantes para os diferentes tipos de aplicações voltadas ao cuidado do idoso. Essa taxonomia contempla 7 tipos de aplicações com 21 categorias e 87 características. A avaliação da taxonomia foi feita com profissionais de saúde e forneceu indícios de que a mesma pode auxiliar a modelagem dos dados para aplicações de saúde do idoso. Entretanto, a combinação entre tipos de aplicação, categorias e características gera um grande número de possibilidades, dificultando sua utilização no processo de desenvolvimento.

Diante disso, este trabalho propõe uma ferramenta Web chamada Dorsal cujo objetivo é apoiar o uso da taxonomia [Araújo et al. 2020] na geração automatizada de modelos de dados para aplicações (Web e/ou aplicativos móveis) voltadas a saúde e cuidado de idosos. Os modelos de dados são gerados com base no tipo de aplicação e nas categorias de dados requeridas pelo problema.

\section{Sobre a Taxonomia}

A taxonomia [Araújo et al. 2020] citada na Seção 1 foi criada iterativamente a partir do método proposto em [Nickerson et al. 2013] e com base na caderneta Brasileira de Saúde do Idoso [da Saúde 2017], que apresenta os pontos revelantes para o cuidado de idosos.

Dentre os 7 tipos de aplicações contempladas pela taxonomia temos: Applications to assist older adult care [Woll and Bratteteig 2019], que compreendem aplicações voltadas para cuidadores de idosos; (ii) Applications of medical treatment and follow-up [Robbins et al. 2018] usadas no acompanhamento de tratamentos médicos; (iii) Home monitoring healthcare systems [Woll and Bratteteig 2019], que são voltadas para o monitoramento de idosos em ambientes específicos como a casa; (iv) Selfcare application [Subramaniam et al. 2016], aplicações criadas para idosos independentes que podem realizar ações de auto-cuidado; (v) Epidemiological clinic systems [Oliveira et al. 2010], sistemas que auxiliam gestores de saúde no enfrentamento de situações epidemiológicas; (vi) Medical normative systems sistemas para suporte de ações normativas dos gestores em saúde [Oliveira et al. 2010]; e, finalmente, (vii) Medical governance applications [Robbins et al. 2018], aplicações relacionadas ao gerenciamento de sistemas de saúde.

As 21 categorias abordadas pela taxonomia são descritas a seguir. Classification of the older adults regarding dependence identifica para que tipo de idoso a aplicação é direcionada (dependente ou independente); Relation with the reference person identifica que tipo de relação há entre o idoso e seu respectivo responsável; Personal data of the 
older adult registra os dados pessoais do idoso; Socio family information traz informações sobre a família do idoso; Reference person data contém dados pessoais do responsável pelo idoso; e Evaluation of the older adult identifica informações sobre medicamentos utilizados pelo idoso. A taxonomia contém também categorias relacionadas a doenças préexistentes (Disease information), cirurgias (Surgeries), reações adversas a medicamentos (Adverse reactions), nutrição (Nutrition), vulnerabilidades do idoso (Identification of the vulnerable older adult), pressão sanguínea (Blood pressure control), índice de glicemia (Glucose control), vacinação (Vaccination), saúde bucal (Oral health), bem como agendamento de consultas médicas (Scheduling of medical inquiries), informações adicionais de saúde (Additional information), hábitos diários (Life habits), avaliação do ambiente no qual o idoso vive (Environmental assessment), existência de doenças crônicas (Chronic pain identification) e dados sobre ocorrência de quedas (Falls).

As 87 características da taxonomia representam informações essenciais, opcionais ou dispensáveis para cada tipo de aplicação. Por exemplo, a característica "Fonte de Renda" (Source of income), presente na categoria Socio family information, é considerada essencial para aplicações de acompanhamento e tratamento médico (Application of Medical Treatment and Follow-up), opcional para aplicações do tipo Home Monitoring Healthcare System, e dispensável para aplicações de auto-cuidado (Selfcare Application).

\section{Ferramenta Proposta}

A Dorsal objetiva auxiliar o desenvolvimento de aplicações de cuidados em saúde de idosos. Ela fundamenta-se na taxonomia proposta por [Araújo et al. 2020] para criar modelos de representação de dados com base no tipo de aplicação desenvolvida e nas categorias de dados relevantes para essa aplicação. Foi desenvolvida em Javascript e está disponível como um serviço Web no link https://great-ufc.github.io/dorsal.

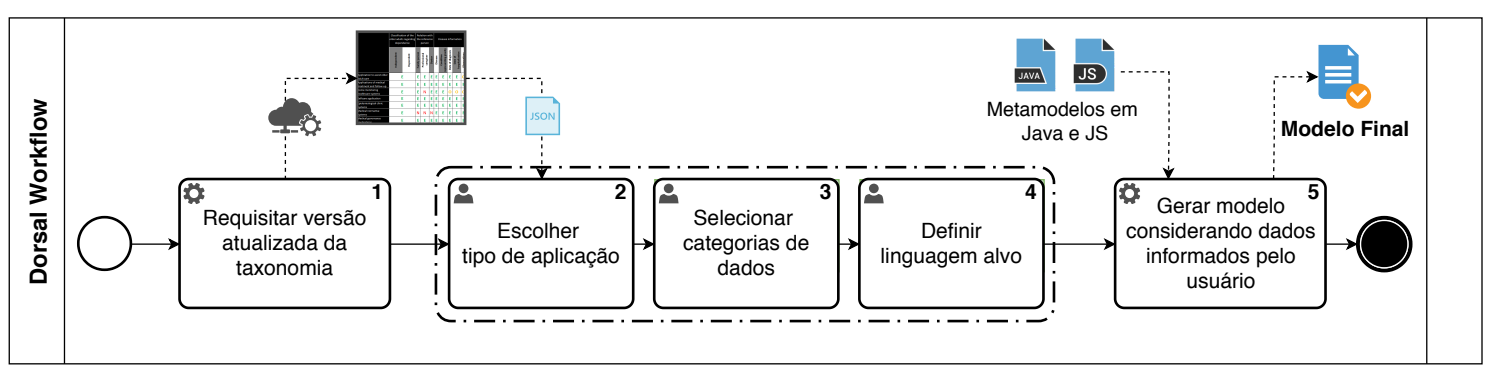

Figura 1. Workflow de utilização da ferramenta Dorsal

A Figura 1 apresenta o workflow da ferramenta. A primeira atividade, realizada automaticamente assim que o usuário acessa a Dorsal, é a requisição de uma versão atualizada da taxonomia. Essa etapa é importante para garantir que a Dorsal reflita possíveis evoluções feitas na estrutura da taxonomia. As atividades 2, 3 e 4 são executadas pelo usuário e consistem, respectivamente, na escolha do tipo de aplicação, das categorias de dados, e da linguagem para a geração do modelo. Atualmente, a Dorsal gera modelos para as linguagens de programação Java e Javascript. No entanto, essa feature pode ser facilmente ampliada com a inclusão de novos metamodelos seguindo o padrão de templates da ferramenta doT.js. Após o acionamento do botão "Export Code", a ferramenta gera um arquivo compactado com o modelo de dados final. 
A combinação entre tipos de aplicação e categorias de dados resulta em características que podem ser essenciais, opcionais ou dispensáveis. As categorias são abstraídas como classes e as características como atributos dessas classes. A Dorsal não inclui as características dispensáveis no processo de geração. Para as opcionais, há uma sinalização por meio de comentários antes do respectivo atributo. Por exemplo, suponha o desenvolvimento de uma aplicação de auto-cuidado ("Self-care application") que necessite armazenar dados pessoais do idoso ("Personal data of the older adult") e informações relacionadas à pessoa de referência deste idoso ("Relation with the reference person"). Características como nome são essenciais. Outras são opcionais (e.g. religião). Tanto essenciais quanto opcionais serão incluídas no modelo. Por sua vez, o nome do cuidador de idoso é dispensável por se tratar de uma aplicação voltada para o auto-cuidado. A Figura 2 apresenta o modelo Java resultante desse exemplo. Assim, o desenvolvedor pode seguir com o processo de codificação atendendo as particularidades da aplicação sob desenvolvimento.

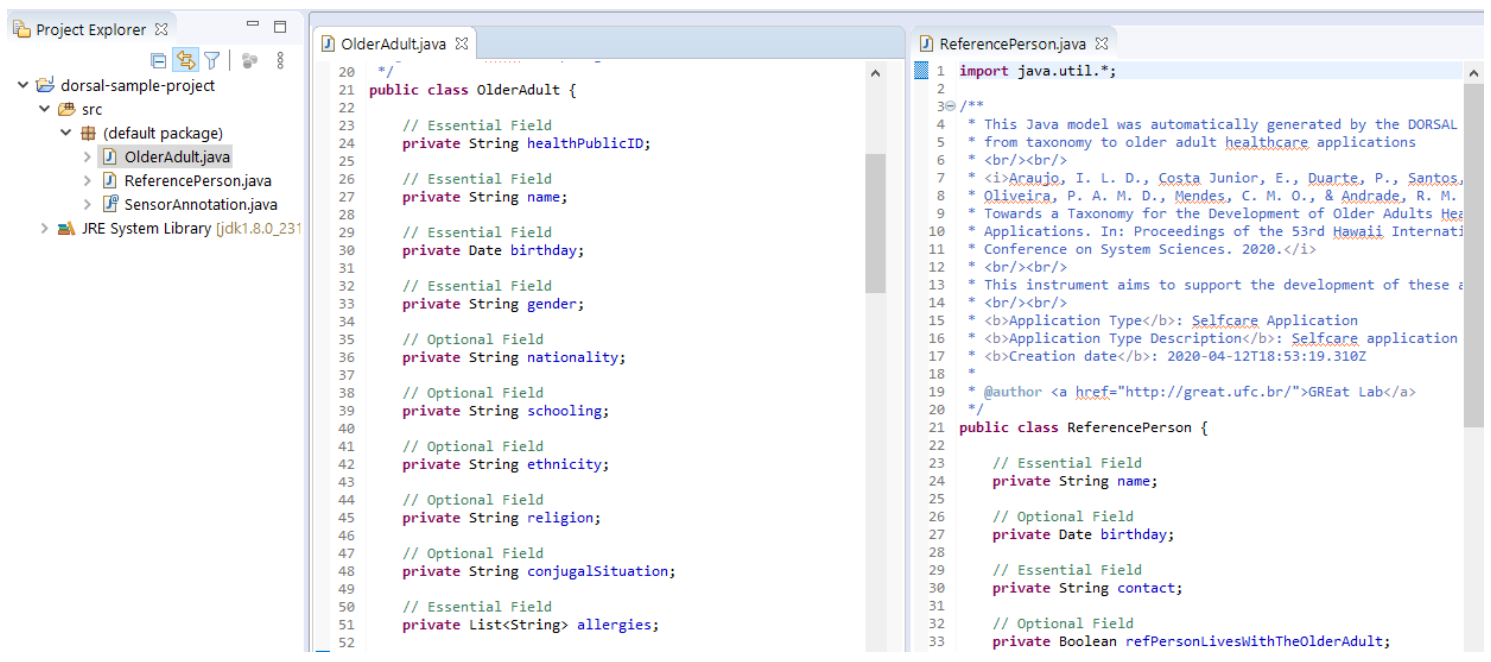

Figura 2. Exemplo de modelo Java gerado pela ferramenta.

\section{Cenário de Uso}

A Dorsal foi projetada de forma a simplificar a construção de modelos de dados a serem utilizados em aplicações voltadas para a saúde do idoso. Para exemplificar possíveis cenários de uso da ferramenta, destacamos três cenários de uso:

I. Desenvolvimento de uma aplicação móvel para a plataforma Android capaz de monitorar os movimentos de idosos a fim de detectar quedas, similar à aplicação WatchAlert [Almeida et al. 2016]. Essa aplicação deve criar um perfil com dados pessoais do idoso, além de registrar a data, local, fraturas e outras consequências sempre que uma queda for detectada.

II. Construção de uma aplicação multiplataforma voltada para a normatização de sistemas em saúde similar a um dos módulos disponibilizados pela ferramenta GISSA 1 Nesse caso, deve-se registrar informações sobre as doenças de determinada população de idosos com o intuito de auxiliar as decisões quanto à normatização de medidas de combate às doenças mais frequentes.

${ }^{1}$ GISSA: https://avicena.in/sobre-o-gissa/ 
III. Desenvolvimento de uma aplicação Web para auxiliar os gestores de saúde no enfrentamento da pandemia do novo coronavírus com foco no grupo de risco das pessoas idosas. Nesse caso, deve-se registrar o maior número de informações possíveis a fim de gerar relatórios que auxiliem o processo de tomada de decisão.

Para o desenvolvimento desses sistemas, é necessária a modelagem de dados. Utilizando a Dorsal, os desenvolvedores podem criar um modelo selecionando o tipo de aplicação, as categorias de dados para atender aos requisitos, e a linguagem alvo. Tais escolhas devem ser fundamentadas nas recomendações da taxonomia e especificidades do sistema sob desenvolvimento. A Tabela 4 apresenta possíveis decisões.

No caso do cenário I, por se tratar de uma aplicação de monitoramento de quedas, o tipo de aplicação deve ser Home Monitoring Healthcare System. Quanto às categorias de dados, deve-se selecionar Personal data of the older adult para registro dos dados pessoais do idoso, e a categoria Falls para registro das quedas. Por fim, a aplicação deve se adequar à plataforma Android. Assim, pode-se utilizar um modelo de dados em Java.

\begin{tabular}{|c|c|c|c|}
\hline \multirow[b]{2}{*}{ Cenários } & \multicolumn{3}{|c|}{ Possíveis Decisões } \\
\hline & Tipo de Aplicação & Categorias de Dados & Linguagem \\
\hline I. & $\begin{array}{l}\text { Sistema de Monitoramento em Saúde } \\
\text { (Home Monitoring Healthcare System) }\end{array}$ & $\begin{array}{l}\text { - Dados Pessoais do Idoso } \\
\text { (Personal data of the older adult) } \\
\text { - Quedas (Falls) }\end{array}$ & Java \\
\hline II. & $\begin{array}{l}\text { Sistema Normativo Médico } \\
\text { (Medical Normative System) }\end{array}$ & $\begin{array}{l}\text { - Dados Pessoais do Idoso } \\
\text { (Personal data of the older adult) } \\
\text { - Informações de Doenças } \\
\text { (Disease information) }\end{array}$ & Javascript \\
\hline III. & $\begin{array}{l}\text { Sistema de Governança Médica } \\
\text { (Medical Governance System) }\end{array}$ & - Todas as 21 categorias & Javascript \\
\hline
\end{tabular}

Para o cenário II, o tipo de aplicação adequado é Medical Normative System. Para as categorias de dados, o desenvolvedor pode escolher Personal data of older adult e Disease information. Destaca-se que, diferente do cenário I, alguns campos para registro de dados pessoais do idoso não serão incluídos, como o nome do idoso. Isso deve-se à necessidade de manter o sigilo de dados pessoais em sistemas de normatização médica. Por fim, como se trata de uma aplicação multiplataforma, optou-se por um modelo em Javascript que pode ser utilizado em frameworks cross-platforms como o React Native².

No cenário III, o tipo de aplicação é Medical Governance System, pois o objetivo do sistema é prover a maior quantidade de dados possíveis aos gestores de saúde. Também por essa razão, o desenvolvedor pode optar por incluir todas as categorias. Ressalta-se que sem o suporte da Dorsal, a modelagem dos dados para esse cenário seria custosa. Por fim, como se trata de uma aplicação Web, pode-se utilizar a linguagem Javascript.

Os modelos gerados para esses cenários (I, II e III) são apresentados no vídeo demonstrativo da ferramenta disponível em https://great-ufc.github.io/dorsal/video. Além disso, é possível acessar o repositório do projeto em https://github.com/great-ufc/dorsal.

\footnotetext{
$\sqrt[2]{\text { React Native: https://reactnative.dev }}$
} 


\section{Considerações Finais}

A Dorsal é uma ferramenta de auxílio à construção de aplicações para saúde de idosos. Com base na taxonomia proposta por [Araújo et al. 2020], a Dorsal gera modelos de representação de dados. Assim, a ferramenta busca reduzir o esforço no desenvolvimento dessas aplicações, além de aumentar a confiabilidade quanto à modelagem de dados.

Como trabalhos futuros, pretende-se realizar um experimento controlado para avaliar o impacto da ferramenta no tempo e na qualidade de desenvolvimento dessas aplicações. Com base nos resultados obtidos, será possível um aperfeiçoamento da Dorsal. Outro ponto de investigação é a integração com middleware de aquisição de contexto que possam semi-automatizar o preenchimento dos modelos gerados.

\section{Referências}

[Almeida et al. 2016] Almeida, R. L., Macedo, A. A., de Araújo, Í. L., Aguilar, P. A., and Andrade, R. M. (2016). Watchalert: Uma evolução do aplicativo falert para detecção de quedas em smartwatches. In Anais Estendidos do XXII Simpósio Brasileiro de Sistemas Multimídia e Web, pages 124-127. SBC.

[Araújo et al. 2020] Araújo, Í. L. D., Costa Junior, E., Duarte, P., Santos, I. D. S., Oliveira, P. A. M. D., Mendes, C. M. O., and Andrade, R. M. D. C. (2020). Towards a taxonomy for the development of older adults healthcare applications. In Proceedings of the 53rd Hawaii International Conference on System Sciences.

[da Saúde 2017] da Saúde, M. (2017). Caderneta de saúde da pessoa idosa - $4^{\circ}$ edição. http://portalarquivos2.saude.gov.br/images/pdf/2017/ setembro/27/CADERNETA-PESSOA-IDOSA-2017-Capa-miolo.pdf.

Accessed: 2019-06-01.

[Nickerson et al. 2013] Nickerson, R. C., Varshney, U., and Muntermann, J. (2013). A method for taxonomy development and its application in information systems. European Journal of Information Systems, 22(3):336-359.

[Oliveira et al. 2010] Oliveira, M., Hairon, C., Andrade, O., Moura, R., Sicotte, C., Denis, J., Fernandes, S., Gensel, J., Bringel, J., and Martin, H. (2010). A context-aware framework for health care governance decision-making systems: A model based on the brazilian digital tv. In 2010 IEEE International Symposium on "A World of Wireless, Mobile and Multimedia Networks” (WoWMoM), pages 1-6. IEEE.

[Robbins et al. 2018] Robbins, T. D., Keung, S. N. L. C., and Arvanitis, T. N. (2018). Ehealth for active ageing: A systematic review. Maturitas, 114:34-40.

[Subramaniam et al. 2016] Subramaniam, S., Dhillon, J. S., Ahmad, M. S., WS LJ, T. C., and Huang, C. (2016). Eliciting user requirements to design a prediabetes self-care application: a focus group study with prediabetics and diabetics. Indian journal of science and technology, pages 1-8.

[WHO 2007] WHO, W. H. O. (2007). Global report on falls prevention in older age. http://www.who.int/ageing/publications/Falls_ prevention7March.pdf. (Accessed on 10/24/2017).

[Woll and Bratteteig 2019] Woll, A. and Bratteteig, T. (2019). A trajectory for technologysupported elderly care work. Computer Supported Cooperative Work, pages 127-168. 